\title{
PURPOSE OF USING CUBESAT SATELLITE TECHNOLOGIES IN THE MILITARY DOMAIN
}

\author{
Dănuț Turcu ${ }^{1}$ \\ "Carol l" National Defence University \\ Gheorghe Adrian Stan \\ "Carol I" National Defence University
}

\begin{abstract}
The increasing usage of space capabilities in the military domain highly requires the development of Nanosatellite polar constellations. In this paper we will analyze the main features of small satellites constellations that could be useful in military purposes. The Nanosatellite or CubeSat technology aim at miniaturization, high modularity and software defined payload, allowing the functionality of entire military space applications and services portfolio, at major low financial costs, translating its scalability and flexibility into a capability. In addition, the efficient combination between low latency and high throughput bandwidth, integrated in federated mega-constellation architectures, transforms CubeSat satellite networks into resilient platforms for development of advanced technologies (5G, loT), according with the military requirements standards.
\end{abstract}

Keywords: Nanosatellite; CubeSat; military space capabilities; polar orbit; advanced technologies.

\section{INTRODUCTION}

Recognition of outer space as the fifth operational domain at NATO level, since 2019 requires a reconsideration of the approach to the peaceful exploration of outer space, in relation to the intensification of its militarization by many states, belonging to the Euro-Atlantic area, but especially is the behavior of states outside of these organizations. The complexity and high financial costs necessary for the operationalization of new space programs, i.e., the upgrading of existing space programs, in conjunction with technological evolution and diversification of space multi-domain missions, lead to the inclusion in the design process of multiorbital space infrastructures of technical solutions in the CubeSat field of technologies.

With this paper we aim to analyze the paradigm, in a SWOT approach, whether the design of CubeSat satellite platforms allows the installation of communication, ISR, Earth observation (EO) or SSA capabilities, and whether these satellite systems, organized in low-orbit constellations (LEO) of the earth, lend themselves to applications on the military domain. Another perspective is given by the fact that, currently in Romania, the space field is considered emerging, being explored and exploited predominantly from the perspective of users of commercial, governmental and military space services, rarely emphasized with isolated initiatives for the operationalization and construction of large-scale space projects, where CubeSat technology, in a professional manner, can be a reliable option in the design of spatial architectures focused on specific missions and organizations.

Development of the advanced technology, at military domain cannot avoid the outer space, taking the consideration that space multiorbital constellations are built to ensure resilient space infrastructures, better connection and bandwidth particularly for end-users or emerging technologies and generally for the ground, air, maritime and cyber domain, because the future military conflict, for sure will be conduct even in the space.

\footnotetext{
${ }^{1}$ Corresponding author: danutturcu@gmail.com
} 


\section{CUBESAT STANDARD EVOLUTION}

Although the CubeSat standard was initially used mainly in the field of scientific research, specific to business and university medium, in recent years the fields of applicability, in which CubeSat satellite systems are used, have diversified and become of interest, including the military domain, taking into account the scalable capacity of the satellite platform, to concentrate in a miniaturized constructive solution, size and small mass, generically called unit (U), a diversity of sensors, configured in a versatile payload, adapted to the typically and mission requirements of different organizations.

Theoretically, the CubeSat standard is a nanosatellite technology, often intertwined, which was first developed and implemented in 1998 as part of a project "at the Aeronautics and Astronautics Department that Stanford University had with DARPA and the Aerospace Corporation, wanted to launch a little satellite, named picosat" (Cappelletti 2021). The term "nanosatellite is usually applied to the name of an artificial satellite with a wet mass between 1 and $10 \mathrm{~kg}^{\prime 2}$, but the consecration of CubeSat space platforms has been used more widely in the design and launch of small satellites. Thus, the result of miniaturizing the components of an artificial satellite, without omitting the basic components of a classic satellite platform (bus, payload and tracking system), of the CubeSat type, were so impressive that "the standard dimensions establishment for each unit $(U)$ has $10 \times 10 \times 11 \mathrm{~cm}$. The size of the CubeSat can be $1 \mathrm{U}, 2 \mathrm{U}, 3 \mathrm{U}, 6 \mathrm{U}$ or $12 \mathrm{U}$, and typically the weight is less than $1.33 \mathrm{~kg}$ for each 1U" (Mahdi 2018), as is depicted in the Figure 1.

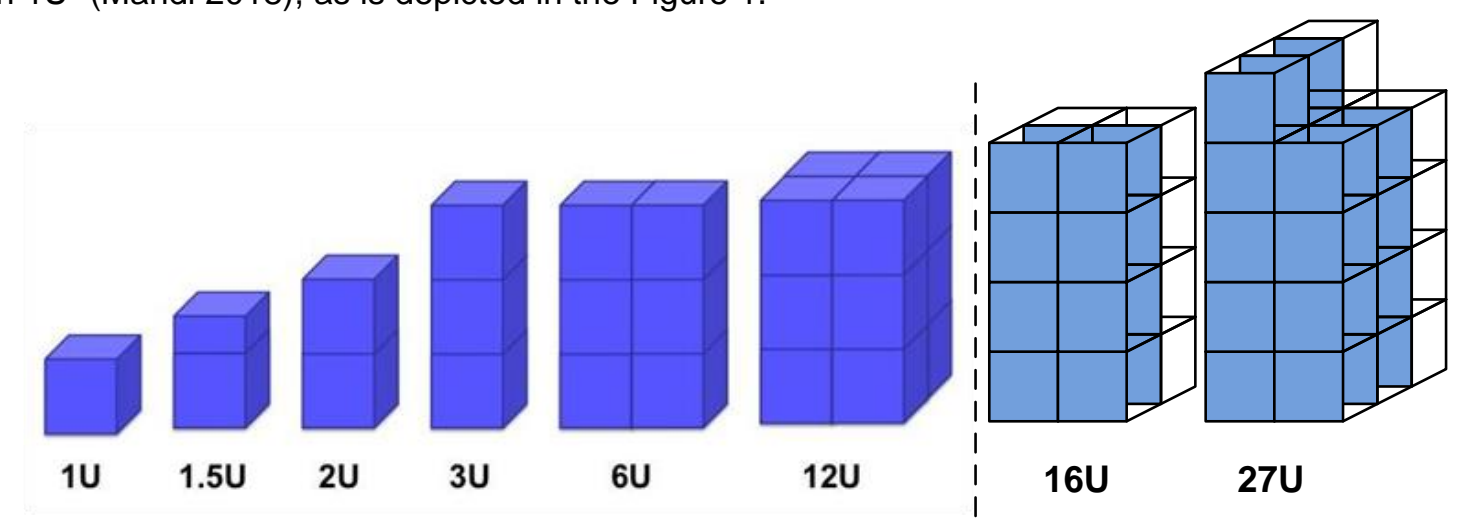

Figure 1. NASA classifications of CubeSat dimensions, adapted ${ }^{\beta}$

Theoretically speaking, CubeSat standard can go further than $12 \mathrm{U}$ dimension as we presented in the Figure 1, of a spacecraft and are possible to discover different classifications, that possible could reach the top limit dimension estimated around $27 \mathrm{U}$ for a CubeSat platform, but practically till now the biggest CubeSat satellite launched reached the dimension of $16 \mathrm{U}$.

According with the ITU-R Report, published in 2014, named "Characteristics, definitions and spectrum requirements of nanosatellites and picosatellites" in order to clarify the issue of small satellite, that started to increase the interest of many organizations and the diversity of applications, regarding the perfect match between size, missions and price, they considered that "satellites weight less than $500 \mathrm{~kg}$ are often referred to as small satellites" (Report ITU-R SA.2312-0 2014) and was classified as shown in Table 1.

These data do not represent the characteristics of an internationally recognized dimensional standard of nanosatellite technology but represent a snapshoot of the technological stage at the time of the analysis, as they are in a continuous upgrading, as a result of technological evolution and the identification of possible new areas of application, in which this kind of space platforms can be useful. According to the classification made by ITU-R, we identify that due to the small size, space platforms in this class, which also include CubeSat space platforms, are limited by:

- duration of operation in space between 1 to 3 years, dependent on the power limits of the bus, as well as the total amount of fuel with which the satellite platform remains on board, after

\footnotetext{
${ }^{2}$ https://nanostarproject.eu/the-project/what-is-a-nanosatellite/, accesed on 08.03.2021.

${ }^{3} \mathrm{https}$ ://www.nasa.gov/content/what-are-smallsats-and-cubesats, accesed on 09.03.2021.
} 
performing the approved orbital operations, applied only for CubeSat satellites that have installed propulsion;

- the specificity of the mission portfolio that such a space platform can execute, depending on the number of sensors that can be installed and configured on the payload;

- operational location exclusively in LEO and HEO polar orbits.

Table 1. Typical characteristics of small satellites (Report ITU-R SA.2312-0 2014)

\begin{tabular}{|c|c|c|c|c|c|c|c|}
\hline Satellite type & $\begin{array}{l}\text { Mass } \\
(\mathrm{kg})\end{array}$ & $\begin{array}{l}\text { Max. } \\
\text { bus } \\
\text { power } \\
\text { (W) }\end{array}$ & $\begin{array}{l}\text { Typical } \\
\text { cost } \\
\text { (USD) }\end{array}$ & $\begin{array}{c}\text { Max. } \\
\text { dimensions } \\
\text { (m) }\end{array}$ & $\begin{array}{c}\text { Development } \\
\text { time } \\
\text { (years) }\end{array}$ & Orbit & $\begin{array}{c}\text { Lifetime } \\
\text { in space } \\
\text { (years) }\end{array}$ \\
\hline Minisatellite & $\begin{array}{l}100- \\
500\end{array}$ & 1000 & $\begin{array}{c}30-200 \\
M\end{array}$ & $3-10$ & $3-10$ & $\begin{array}{l}\text { GEO } \\
\text { MEO } \\
\text { LEO } \\
\text { HEO }\end{array}$ & $5-10$ \\
\hline Microsatellite & $10-100$ & 150 & $10-150 M$ & $1-5$ & $2-5$ & \multirow{4}{*}{$\begin{array}{l}\text { LEO } \\
\text { HEO }\end{array}$} & $2-6$ \\
\hline Nanosatellite & $1-10$ & 20 & $\begin{array}{c}100 \mathrm{k}- \\
10 \mathrm{M}\end{array}$ & $0.1-1$ & $1-3$ & & $1-3$ \\
\hline Picosatellite & $0.1-1$ & 5 & $50 \mathrm{k}-2 \mathrm{M}$ & $0.05-0.1$ & $1-3$ & & $1-3$ \\
\hline Femtosatellite & $<0.1$ & 1 & $<50 \mathrm{k}$ & $0.01-0.1$ & 1 & & $<1$ \\
\hline
\end{tabular}

Moreover, the registration data on the evolution of CubeSat and nanosatellite capabilities, from 1998 onwards and continuing with a calculated forecast until 2025, they have been summarized in the Nanosats database, created and updated periodically, since 2013 by space engineering expert Erik Kulu, a collaborator of Tartu University/Estonia, between 2016 and 2019, for the development of scientific research projects in the field of construction and launching of CubeSat satellites. The accuracy of the input data in this database cannot be maximum, because some organizations do not publicly present their spatial projection, but it is updated quarterly by the author and have as their main basis a variety of sources, such us "launch satellite schedules, outer space stores and articles, official websites, various databases, presentation from related conferences, space reports and master of satellite list"4. Research from this database shows that since 1998 about 2968 space missions with CubeSat and Nanosat platforms have been planned and registered, belonging to a wide range of organizations, including the military organization. Thus, are the most recent update of this database was made in January 2021, resulting in the following data, presented in Table 2 and depicted in Figure 2, as follow:

Table 2. Typical characteristics of small satellites ${ }^{5}$

\begin{tabular}{|c|c|c|c|c|}
\hline $\begin{array}{c}\text { Nanosats } \\
\text { launched }\end{array}$ & $\begin{array}{c}\text { CubeSat } \\
\text { launched }\end{array}$ & $\begin{array}{c}\text { Inmterplanetary } \\
\text { CubeSats }\end{array}$ & $\begin{array}{c}\text { Countries with } \\
\text { nanosats }\end{array}$ & Forecast \\
\hline 1474 & 1357 & 2 & $70^{*}$ & $>2500$ \\
\hline
\end{tabular}

* A special mention refers to Romania regarding space activities. According to this database we could identify three space projects were Romania represented by University of Bucharest, launched in 13.02.2012, first artificial $1 U$ CubeSat satellite, named" GOLIAT", with VEGA rocket, on LEO orbit $(310 \times 1450 \mathrm{~km} / 69,50)$. This satellite was operational for a short time because of lost tracking connection with the ground TT\&C station, caused by the jamming of tracking frequencies. GOLIAT reentered in Earth atmosphere in 31.12.2014. Shortly after this attempted, another space project was launched by a joint venture between Romanian Space Agency (ROSA) and Institute of Space Science (ISS), that planned to launch two $2 U$ CubeSat satellites, named "RoBiSat-1" and "RoBiSat-2"6. Unfortunately, this ambitious space science project was cancelled due lack founding.

\footnotetext{
${ }^{4} \mathrm{https}: / /$ www.nanosats.eu/database, accesed on 10.03.2021.

${ }^{5} \mathrm{https}: / / \mathrm{www}$.nanosats.eu/database, accesed on 10.03.2021.

$6 \mathrm{http}: /$ www2.rosa.ro/index.php/en/space-strategy/programul-star/proiecte-finantate-prin-competitia-c1-2012/98-cd1/385-romanian -dualcubesat-mission-development-of-the-robisat-1-spacecraft, accesed on 10.03.2021.
} 


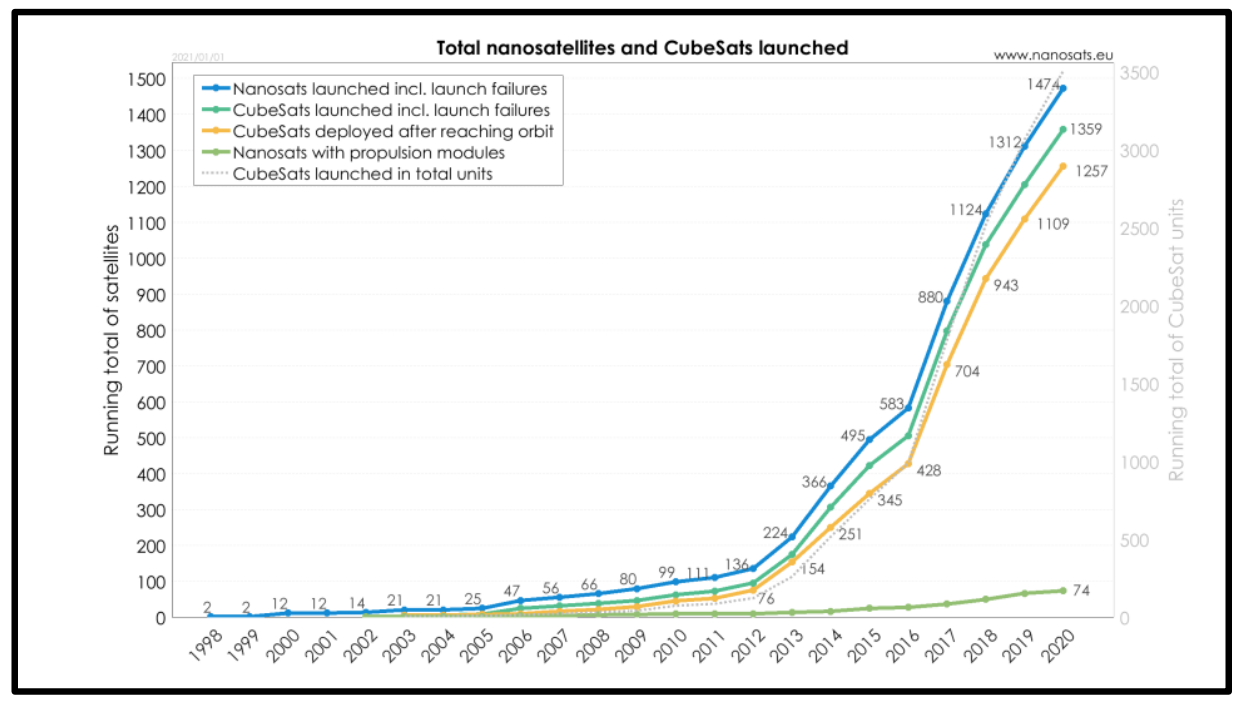

Figure 2. Total nanosatellites and CubeSats launched

Starting with 2013, as is depicted in Figure 2, we can see the tremendous growing of CubeSat/Nanosats missions launched by different organizations, also including the launch failures. Other interesting fact from this database that we can identify is the highly rate, about $6.3 \%$, of small satellites failures during launching procedures and launch vehicle failure. Moreover, the issue of the high failure rate of small satellites projects is also confirmed and deeply analyzed by Stephen A. Jacklin, from NASA Ames Research Center in the study "Small-Satellite Mission Failure Rates". The study reveals the facts that from " 2000 to $2016,41.3 \%$ of all small satellites launched experienced total or partial mission failure. Of these, $6.1 \%$ were launch vehicle failures, $11 \%$ were partial mission failures, and $24.2 \%$ were total mission failures" (Jacklin Stephen 2019). In our opinion an important clue of this failure high percentage could gravity around the problem of avoidance by the organizations, especially by the university projects, the ITU spectrum international coordination process, important and highly time /money consumer stage, in the process of satellite manufactures.

Another important criterion of this database is referring to the affiliation of CubeSat satellite projects by types of organizations, were military CubeSat initiatives published, reached the number of 125 projects, represented $4,2 \%$ from the total number of CubeSat projects, the majority of this being under the development of US DOD. From this value we can identify at list 50 projects that are operational and other 75 projects that could be under development and are not launched yet, as is depicted in Figure 3.

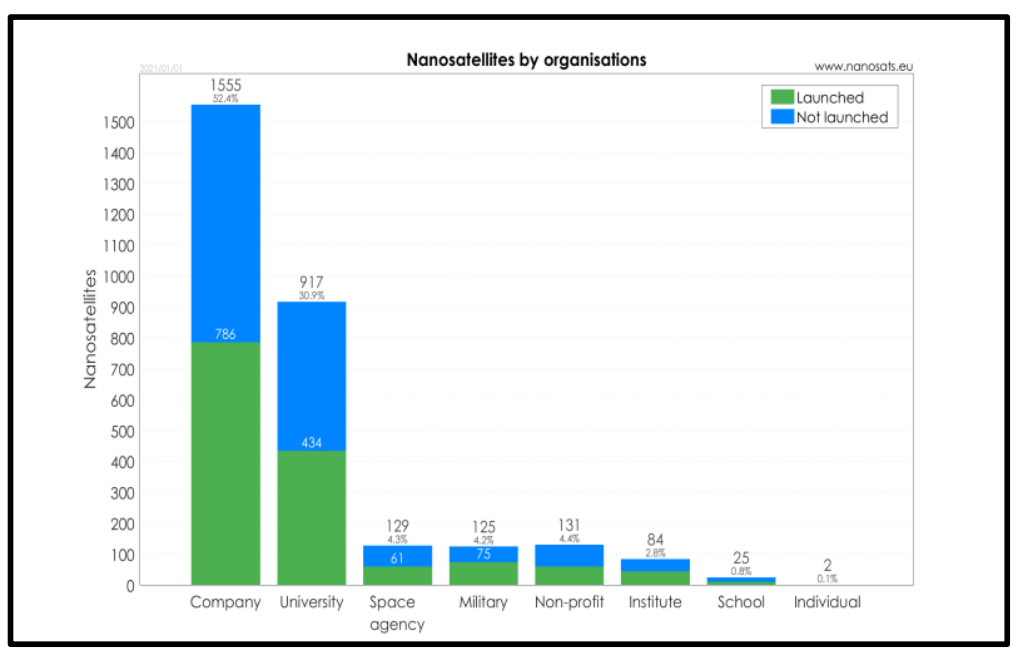

Figure 3. Nanosatellites by organizations ${ }^{8}$

\footnotetext{
${ }^{7} \mathrm{https}: / /$ www.nanosats.eu/database, accesed on 10.03.2021.

${ }^{8} \mathrm{https}: / / \mathrm{www}$. nanosats.eu/database, accesed on 10.03.2021.
} 
Taking in consideration that space is declared warfighting domain, is highly disputed and militarized, in near future we appreciate that many world armies, including small countries, could include the CubeSat LEO constellations as part of the future military space programs, because of their reliability, affordability and flexibility. Other reason is about the possible development and implementations of the advanced technologies, such as $5 \mathrm{G}$ and loT, in military domain using swarm satellite constellations, in order to create a resilient federated critical space infrastructure. This opportunity can establish the link between other warfighting domains, and especially cyber domain, as far space domain and cyber converge each other in various scenarios.

\section{SWOT ANALYSIS}

Military space programs "provide critical information, intelligence, warning, and communication capabilities to commanders and warfighters across the spectrum of global conflict" (Nayak Michael p. 92). In order to identify if the CubeSat technologies is a interesting solution in development of military space programs we will do a SWOT analyze, in order to relieve as many as possible pro and negative arguments, that could be take in considerations by different military stake holders in the beginning of design process.

\section{STRENGTHS}

- can be build in short period of time, about 1-2 years, comparing with large satellite that are built in 3-5 years;

- CubeSat in less expensive comparing with large satellites, that permit to the owner the option on building swarm constellations;

- easy to find launching facilities, even the opportunity of launching the CubeSat from local facilities;

- CubeSat technology is simple, also the design and it's very easy to adapt according to the missions;

- flexible in design of space multiorbital architectures;

- reliable for image, EO and communications, using store\&forward capabilities;

- easy to launch space capabilities for short missions or for typical military applications (Link 16 , satellite communications);

- affordable in ITU coordination frequencies procedure.

\section{WEAKNESSES}

- limited by using only in LEO and HEO orbits;

- short period of mission duration, about $1-2$ years, comparing with the large satellite were the mission duration is estimated at list about 15 years, guaranteed by the producers;

- limited by the dimension of payload, that imply the reduce number of missions. All most the CubeSat platforms is designated for a single mission;

- very difficult to implement on board active and passive protection solutions for this platforms;

- hard or impossible to develop SAR CubeSat platform;

- limited solutions for implementing beam forming and adaptive antennas;

- easy to lost the satellite from tracking.

\section{OPPORTUNITIES}

- to ensure worldwide coverage for different space missions is less expensive;

- reduce quantity or no space debris resulted, because CubeSat platform parts are entirely burned at the ending of lifecycle, when it re-entered in the Earth atmosphere;

- opportunity of including the national space industry in the development of the CubeSat platforms;

- easy to create a national military space programs with small amount of money;

- design flexible architectures including multiorbital space capabilities; 
- create the chance of developing national educational and research programs;

- possibility to test new space solutions and technologies;

- reduce dependency from commercial space service providers;

- provide space services for users in expedition missions;

- opportunity for development emerging technologies such is blockchain, 5G and loT;

- possibility to saw in real time the enemy troops movement and to actions according with their actions;

- increase the accuracy of monitoring the space as a part of Space Situational Awareness (SSA) and Space Traffic Management (STM) projects;

- provide better connections for users, according to the principles of low latency and high throughput bandwidth, transforming the space in a "optical fibre";

- possibility of including SDR solutions on CubeSat platform;

- easy to transform the satellite in a potential ASAT;

- "develop offensive and defensive counterspace operations" (Galliand Chalie);

- can provide the evolution of space domain.

\section{THREATS}

- large number of CubeSats launcher are failure;

- easy to jam the tracking, and communications frequencies of the satellite;

- simple to destroy this satellite with ASAT capabilities;

- high possibility for collisions with space debris;

- vulnerability of cyber-attackers and electronic warfare actions;

- possibility of losing the satellites during the mission, because of life length;

- small CubeSat constellation increase the chance of lost connections between users and the coverage can be a issue;

- information can be compromised and un-useful for users, because of the reduce number of satellites, and it's refers to period of time past since the moment when data is collected from space till arrived to users.

\section{CONCLUSIONS}

From the analysis carried out, we find that CubeSat technology favors the development of spatial programs applicable in the military field, taking into account adaptability to missions, low financial costs of achievement and the opportunity to project the national interests of the state in space. The negative elements of CubeSat technology gravity around the fragility of these satellite systems, high vulnerability to possible attacks by the enemy, and last but not the list, small size of these satellite platforms show limitations in the design of space missions. CubeSat capabilities promote space research, education and testing of new solutions.

According to growing military interest in small satellites, the future military space programs, CubeSat capabilities can only present the possible solutions in developing a multiorbital architecture, by exploiting both advantages of GEO satellites and small satellites operated in the MEO and LEO orbits. They are also active components of the SSA and STM programs, fundamental to the process of management of space infrastructures.

To summarize this paper the Nanosatellite or CubeSat technology in military applications aim the miniaturization, high modularity and software defined payload, allowing the functionality of entire military space applications and services portfolio, at major low financial costs, translating its scalability and flexibility into a capability. In addition, the efficient combination between low latency and high throughput bandwidth, integrated in federated mega-constellation architectures, transforms CubeSat satellite networks into resilient platforms for development of advanced technologies, according with the military requirements standards. 


\section{REFERENCES}

Cappelletti, Chantal, Simone Battistini, Benjamin K. Malphrus. 2021. Cubesat Handbook from mission design to operations.

Galliand, Chalie, Study of the small: Potential for operational military use of CubeSat - presentation, US Space Test Program.

Jacklin, Stephen A. 2019. Small-Satellite Mission Failure Rates, NASA Ames Research Center in the study.

Mahdi, Mohammed Chessab. 2018. Attitude Stabilization for Cubesat Concepts and Technology, Cambridge Scholars Publishing, p.1.

Nayak, Michael Capt., Deterring Aggressive Space Actions with Cube Satellite Proximity Operations A New Frontier in Defensive Space Control, Air \& Space Power Journal, p.92.

REPORT ITU-R SA.2312-0. 2014. Characteristics, definitions and spectrum requirements of nanosatellites and picosatellites, as well as systems composed of such satellites, Electronic Publication Geneva, p. 2-3.

https://nanostarproject.eu/the-project/what-is-a-nanosatellite/, accesed on 08.03.2021.

https://www.nasa.gov/content/what-are-smallsats-and-cubesats, accesed on 09.03.2021.

https://www.nanosats.eu/database, accesed on 10.03.2021.

http://www2.rosa.ro/index.php/en/space-strategy/programul-star/proiecte-finantate-prin-competitia-c12012/98-cd1/385-romanian-dual-cubesat-mission-development-of-the-robisat-1-spacecraft, accesed on 10.03.2021. 